\title{
Visualisierung analoger Schaltungen durch 3-D Animation von transienten SPICE-Simulationen
}

\author{
J. Becker and Y. Manoli \\ Universität Freiburg, Institut für Mikrosystemtechnik - IMTEK, Lehrstuhl für Mikroelektronik, Freiburg, Germany
}

\begin{abstract}
Zusammenfassung. Für das Zeichnen analoger Schaltpläne wird oft versucht, die Potentialverteilung in der entsprechenden Schaltung auszunutzen, um eine Platzierung der Bauteile nach abfallendem Potential vorzunehmen. Mit Hilfe von Computerunterstützung gelingt es, eine verallgemeinerte dreidimensionale Platzierungsstrategie anzuwenden, die allein auf Grund der Potentialwerte einer Schaltung die automatische Generierung einer technisch exakten Potentialdarstellung erlaubt. Somit ist es möglich, die Ergebnisse von transienten SPICE-Simulationen in jedem Zeitschritt darzustellen und eine Animation des zeitlichen Verhaltens zu erzeugen. Die Umsetzung dieser Methode zur Einbettung in eine webbasierte Lern - und Arbeitsplattform wird im Folgenden erläutert.
\end{abstract}

\section{Einleitung}

Durch die stetig zunehmende Präsenz von Computern in Forschung und Lehre ist es nunmehr selbstverständlich, dass Vorlesungen und Kurse über elektronische Schaltungen durch die Benutzung von Simulationsprogrammen bereichert werden. Diese sind meist auf die Benutzung durch Experten in professionellen Bereichen ausgerichtet und übersteigen weit sowohl die Anforderungen als auch den Aufwand, der für eine reibungslose Nutzung in der Lehre angemessen ist. Darüber hinaus werden Ergebnisse von Transientsimulationen üblicherweise als waveform-plots der Spannungsverläufe dargestellt, welche unter Umständen schwierig zu interpretieren sind. Der Benutzer muss eine Abstraktion der Schaltung entwickeln, indem er ein analytisches Verständnis der Spannungsverläufe mit einem graphischen Verständnis des Schaltplans vereint, was zu subjektiven und teilweise falschen Modellbildungen beim Lernenden führt. Um ins-

Correspondence to: J. Becker

(jmbeck@imtek.de) besondere Neulinge im Gebiet der elektronischen Schaltungen von Anfang an bei der Bildung eines wissenschaftlich korrekten Verständnisses zu unterstützen, wird im folgenden die Darstellungsmethodik nach Becker and Manoli (2006b) vorgestellt, mit der es durch so genannte potentialäquivalente Platzierung gelingt, Strom/Spannungsdarstellung und Schaltplan in einer einheitlichen Abstraktion konsistent zu vereinen.

\section{Darstellungsmethodik}

Durch den Einsatz moderner Computergraphik ist es in einer interaktiven Lernumgebung möglich, analoge Schaltungen in einem zweidimensionalen Editor einzugeben und diese mit zusätzlichen Informationen für eine Platzierung im dreidimensionalen Raum zu versehen. Dabei wird die Position eines Bauteils wie in Abb. 1 durch eine Koordinate auf der (x,y)-Ebene vom Benutzer festgelegt.

Abbildung 2 verdeutlicht die Methode der potentialäquivalenten Platzierung, bei der Simulationswerte der Spannungen innerhalb einer Schaltung benutzt werden, um Netze auf einem vertikalen Potentialraster einzuordnen und die Bauteile dazwischen zu skalieren. Netze sind per Definition in der Simulation als ideale Leiter angenommen und somit isopotential. Damit erscheinen sie in einer solchen Darstellung immer als horizontale Linien oder Punkte. Über Bauteilen hingegen besteht eine Spannung, die als die Differenz der angeschlossenen Potentiale sofort graphisch als Höhe sichtbar wird.

Abbildung 3 zeigt eine Beispielschaltung im Editor der Lernsoftware, wobei hier sowohl Bauteile als auch Netzpunkte ( $p 01, p 02$ und gnd) individuell verschiebbar sind. Die $\mathrm{x}$-Koordinate wird durch die Platzierung auf einer horizontalen Spalte bestimmt und die y-Koordinate durch eine Eingabe der Reihennummer in den viereckigen Markern an jedem Objekt. Reihe $O$ ist die Grundeinstellung und Objekte können entweder in positiver Richtung (aus der

Published by Copernicus Publications on behalf of the URSI Landesausschuss in der Bundesrepublik Deutschland e.V. 


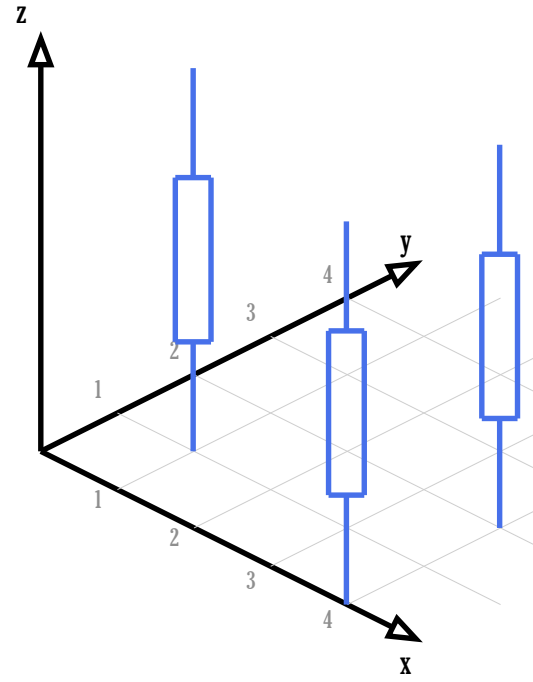

Abb. 1. Anordnung von Bauteilen in x-Spalten und y-Reihen.

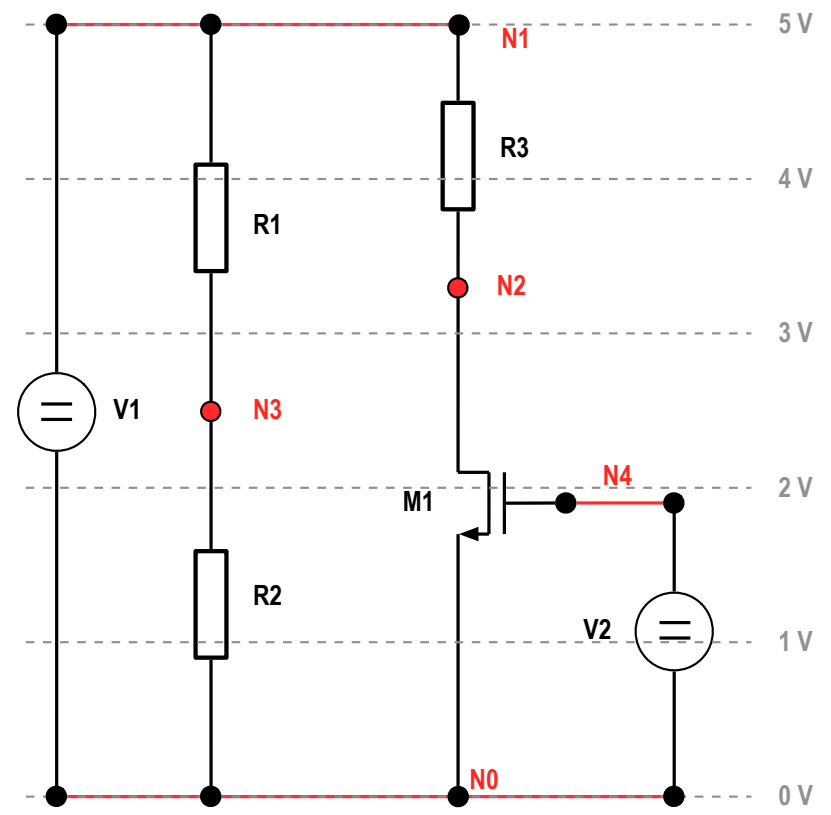

Abb. 2. Beispiel einer potentialäquivalenten Darstellung.

Zeichenebene heraus) oder in negativer Richtung (in die Zeichenebene hinein) um ganze Zahlenwerte verschoben werden. Abbildung 4 zeigt eine aus diesen Angaben berechnete 3D-Ansicht des gleichen Schaltplans, wobei zu erkennen ist, dass das Voltmeter $x 01$, Netzpunkt $p 02$ und die Spannungsquelle vin in der gleichen Reihe liegen, der Kondensator $\mathrm{cO1}$ samt Netzpunkt $p 01$ aus der Zeichenebene hervortreten, und Widerstand $r 01$ und Netzpunkt gnd um eine Reihe nach hinten verschoben sind.

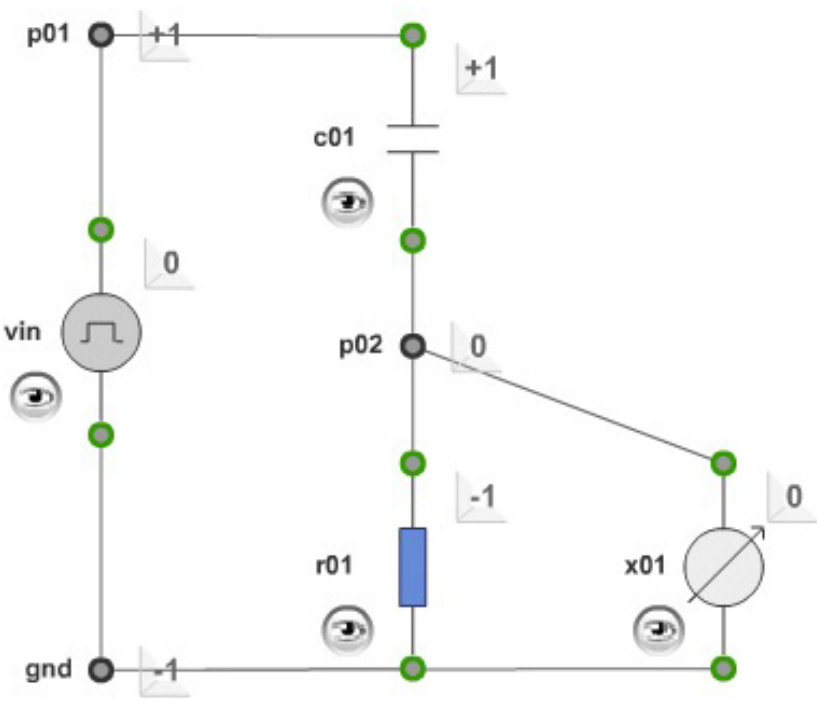

Abb. 3. Bauteilplatzierung im 2D-Editor.

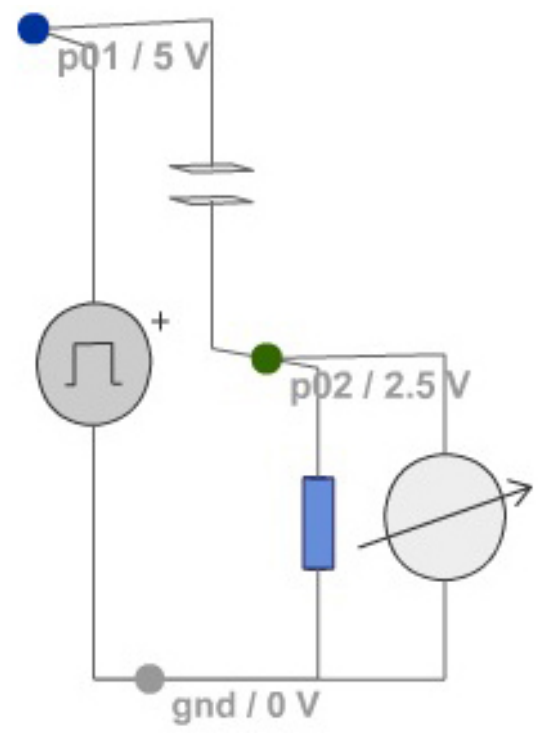

Abb. 4. 3D-Darstellung des Schaltkreises in der Animation.

Die Anordnung der Objekte in z-Richtung, also der Höhe, erfolgt wiederum durch potentialäquivalenten Platzierung. Netz p02 spannt in Abb. 4 zwischen den Verbindungsstellen der angeschlossenen Bauteile eine Potentialebene auf, die im gezeigten Beispiel auf der $2.5 \mathrm{~V}$ zugeordneten Höhe liegt. Um Netzpunkt p01 herum deutet die Ecke von Reihe +1 zu Reihe 0 die Potentialebene für $5 \mathrm{~V}$ an, während gnd mit $0 \mathrm{~V}$ die Referenzfläche markiert. Bei einer planen Projektion der 3D-Darstellung auf die Zweidimensionalität der Abb. 4 müssten $c 01$ und $r 01$ senkrecht untereinander stehen, da sie 


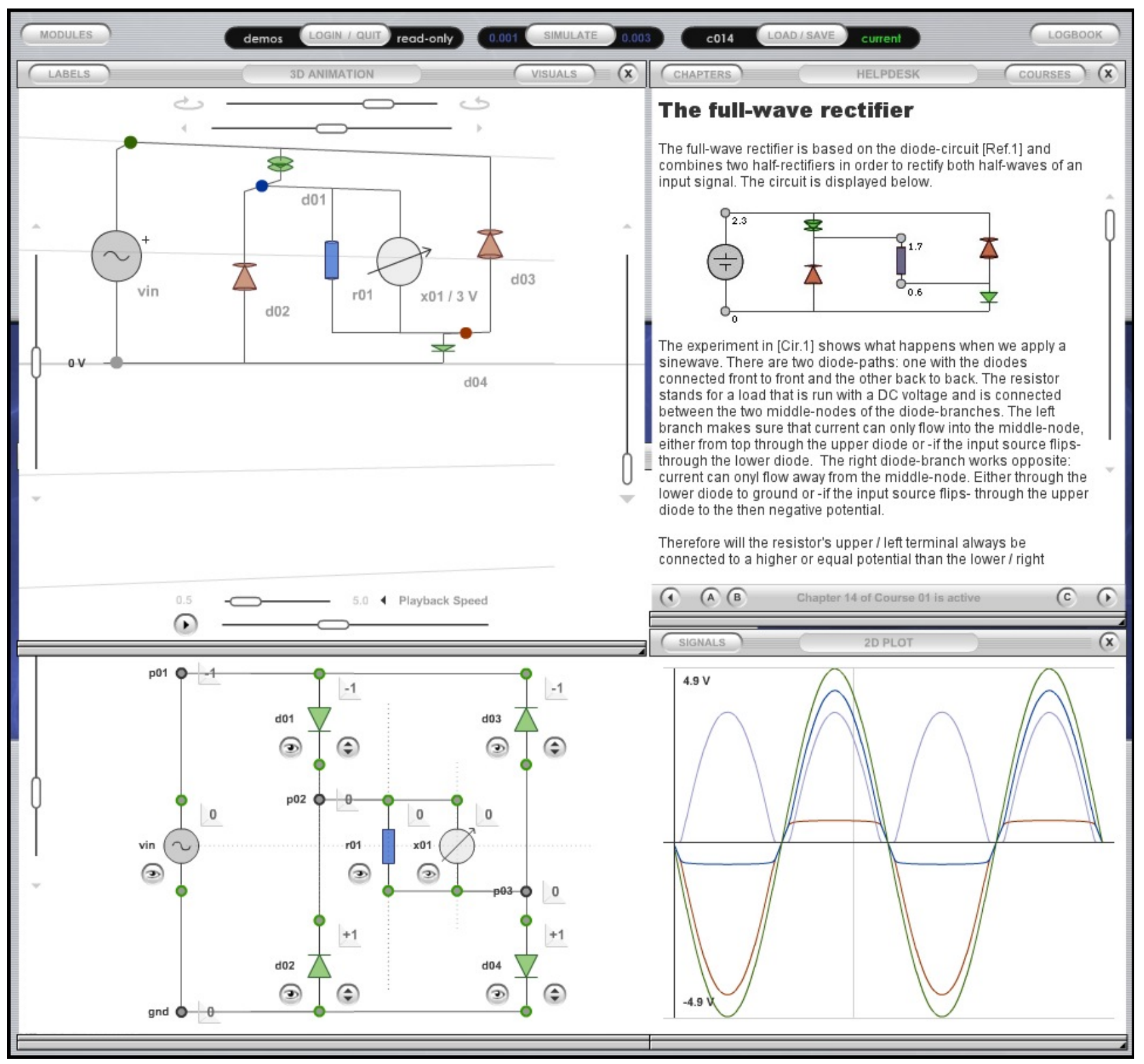

Abb. 5. Übersicht über die gesamte Oberfläche der Lernsoftware.

in Abb. 3 in der selben Spalte platziert wurden. Der Schaltplan in Abb. 4 erscheint hier leicht im Uhrzeigersinn um die Mittelachse gedreht, damit die Räumlichkeit für den Betrachter verständlich wird. In der interaktiven Lernsoftware ist es durch Computerunterstützung möglich, dem Benutzer eine freie Wahl des Betrachtungswinkels zu geben, wodurch das Verständnis der Darstellung sehr vereinfacht wird.

\section{Animationsdarstellung}

Abbildung 5 zeigt eine Übersicht über die gesamte graphische Benutzeroberfäche der Lernsoftware, wie sie nach Becker and Manoli (2006a) umgesetzt wurde. Alle Module der Anwendung, wie Editor, 3D-Animation, 2D-Plot und andere sind in separaten Fenstern implementiert, die frei verschoben und skaliert werden können. Links unten ist ein Ausschnitt des Editors zu sehen, in dem der Benutzer Schaltungen nahezu beliebiger Komplexität frei verdrahten kann. Als Bauteile stehen bisher zur Verfügung: 


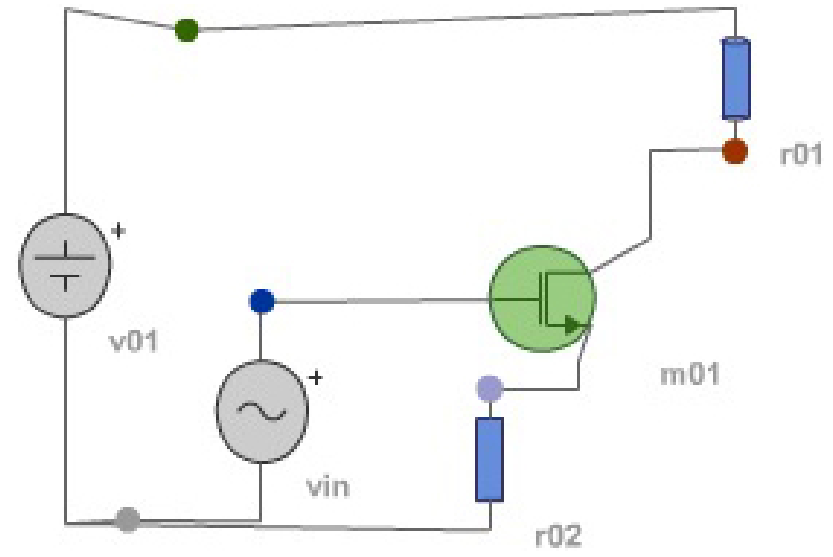

Abb. 6. Transistorverstärker im Arbeitsbereich.

Strom- und Spannungsquellen, gesteuerte Quellen, Widerstände, Kondensatoren, Dioden, Bipolar-Transistoren, MOS-Transistoren und zur Anzeige von Spannungen Voltmeter. Durch die Verwendung des de-facto Standards SPICE für die Simulation, sind die Möglichkeiten der verwendbaren Bauteile leicht erweiterbar auf alle Arten, die durch SPICEModelle beschrieben werden können. Das System ist somit nicht beschränkt auf den jetzigen Funktionsumfang sondern kann mit den Anforderungen der Benutzer wachsen.

Für die aktiven Bauteile, denen in SPICE Modellbeschreibungen für die Simulation hinzugefügt werden können, existiert ein Eingabefenster zur Verwaltung der Modelle. So kann falls gewünscht jedem Bauteil ein individuelles Simulationsmodell beliebiger Komplexität zugeordnet werden, wie es von SPICE bekannt ist.

Abbildung 5 rechts unten zeigt den Verlauf der Spannungen innerhalb der Schaltung, wie sie nach einer Transientsimulation traditionell in einem waveform-plot dargestellt werden. Die Besonderheit der vorgestellten Software besteht nun darin, dass für jeden Zeitschritt der Simulation die Potentiale den entsprechenden Bauteilen zugeordnet werden und aus der Abfolge von daraus generierten 3D-Ansichten ein zeitlich animierter Film entsteht. Dieser kann im Animationsfenster (hier links oben) gesteuert und betrachtet werden. Über die Regler an den Fensterkanten können Zoom, Verschiebung und Betrachtungswinkel stufenlos eingestellt werden, wodurch der Betrachter eine räumliche Wahrnehmung der Schaltung erhält. Ein weiterer Regler bestimmt den Zeitpunkt der Simulation, der dargestellt werden soll und dieser wird auch durch eine Haarlinie im 2D-Plot angezeigt. Durch die gleiche Farbgebung der Kurven und Netzknoten wird eine der Zusammenhang zwischen diesen beiden Darstellungen verdeutlicht.

Eine automatische Abspielfunktion schließlich erlaubt die flüssige Animation der 3D-Ansicht im zeitlichen Ablauf, wodurch die Funktionsweise der Schaltung durch Bewegun-

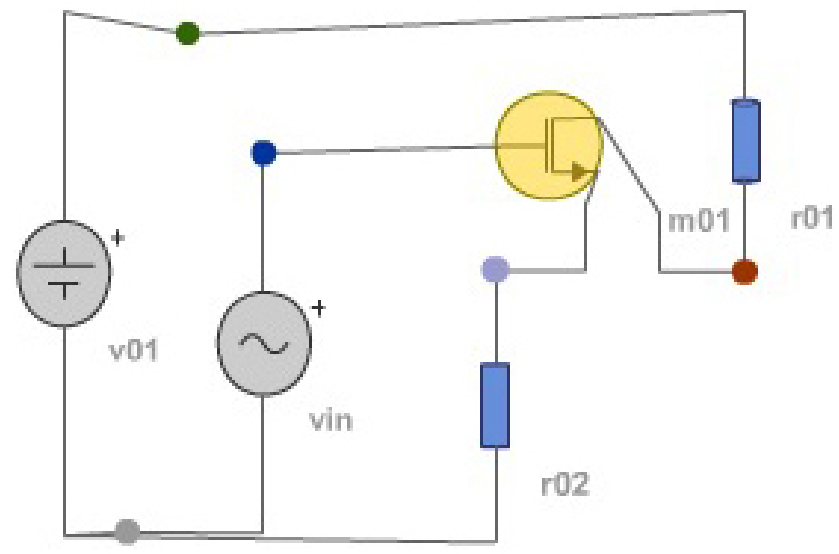

Abb. 7. Transistorverstärker ausserhalb des Arbeitsbereiches.

gen der Bauteile in der potentialäquivalenten Darstellung verständlich wird. Um das Verständnis von Wirkprinzipien $\mathrm{zu}$ unterstützen ist es außerdem möglich, die Operationspunkte von aktiven Bauteilen farblich zu kennzeichnen. Dioden können je nach Stromrichtung grün (leitend) oder rot (sperrend) dargestellt werden und Transistoren rot (aus), gelb (linear), grün (Sättigung). Wenn beispielsweise ein Transistorverstärker wie in Abb. 6 an die Grenzen seines dimensionierten Arbeitsbereiches ausgesteuert wird, kann beobachtet werden wie gleichzeitig der Transistor die Farbe wechselt und eine Verzerrung des Ausgangssignals auftritt. Ein Vergleich mit Abb. 7 zeigt die Farbänderung des Transistors und die Potentialänderung der Netze. Statische Bilder können hier leider nur sehr rudimentär wiedergeben, wie eine flüssige Animation wirkt, und ein wesentlicher Bestandteil der Perzeption ist sicherlich der interaktive Umgang mit der Applikation.

\section{Hilfestellungen}

Neben der eigentlichen Applikation zur Darstellung von Schaltplänen beinhaltet die Lernplattform auch ausführliche Hilfestellungen. Es existiert eine reiche Anzahl von lehrbuchartigen Artikeln, die in einem eigenen Fenster dargestellt werden (Abb. 5, rechts oben) und thematisch aufeinander aufbauen. $\mathrm{Zu}$ jedem Themengebiet können vordefinierte Schaltplän geladen, animiert und eigenständig verändert werden. Durch interaktives Ausprobieren und die Möglichkeit, vorhandene Schaltungen mit eigenen Ideen zu bereichern, wird ein spielerisches Lernen und Experimentieren gefördert. Die Speicherung der erstellten Schaltungen auf dem Web-Server ermöglicht den einfachen Austausch zwischen Studenten und somit ein ortsunabhängiges Zusammenarbeiten und Diskutieren. 


\section{Diskussion}

Es wurde eine Lernsoftware vorgestellt, die eine neuartigen Darstellungsmethode für analoge Schaltungen implementiert. Durch gleichzeitige Visualisierung von Spannungen durch potentialäquivalente Platzierung und Operationsbereichen durch Farben entsteht eine Hervorhebung der Funktionsprinzipien elektrischer Schaltungen. Die Animation von Simulationsergebnissen liefert zudem eine Abstraktion der elektrischen Wirkprinzipien, die wesentlich zum Verständnis, vor allem auch bei Anfängern, beitragen kann.

\section{Literatur}

Becker, J. and Manoli, Y.: An Online Learning-Environment with Embedded 3D Visualization and Animation of Electronic Circuits, World Conference on E-Learning in Corporate, Government, Healthcare, and Higher Education (eLearn), 2006a.

Becker, J. and Manoli, Y.: Computer-aided 3D animation of analog circuits, 6th European Workshop on Microelectronics Education (EWME), 2006b. 